\title{
MOBILIDADE DO HERBICIDA ATRAZINA EM AMOSTRAS DE LATOSSOLOS VERMELHOS SOB CALAGEM E ADUBAÇÃO FOSFATADA
}

\author{
FERNANDA CARLA WASNER VASCONCELOS* \\ JÚLIO CÉSAR AZEVEDO NÓBREGA** \\ MÁRIO CÉSAR GUERREIRO*** \\ ELEN ALVARENGA DE JESUS ${ }^{* * * *}$

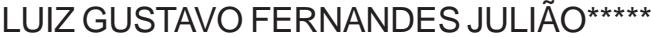 \\ NILTON CUR| $\left.\right|^{\star \star \star \star \star \star}$
}

O objetivo deste estudo foi avaliar o efeito das práticas de calagem e adubação fosfatada, isoladamente ou em conjunto, sobre a lixiviação da atrazina em amostras de Latossolos Vermelhos com teores diferenciais de óxidos de ferro (em lisímetros) sob condições de laboratório. Os tratamentos foram dispostos em delineamento experimental inteiramente casualizado, esquema fatorial $2 \times 2$, sendo testados os seguintes fatores: solos em duas condições de fósforo (com e sem fosfato) e duas condições de calagem (com e sem calagem), com três repetições, totalizando doze parcelas experimentais para cada solo. A atrazina foi aplicada em dose correspondente a $6 \mathrm{~L} \mathrm{ha}^{-1}$ de Gesaprim $500^{\circledR}$, sendo simuladas chuvas de $18,75 \mathrm{~mm}$ a cada três dias durante o período de 30 dias. O material amostrado sólido foi devidamente preparado para extração da atrazina e quantificado em cromatógrafo a gás, equipado com detector de nitrogênio e fósforo (CG-NPD). Os resultados obtidos mostraram que, independentemente dos solos e práticas de calagem e adubação fosfatada, a atrazina apresenta mobilidade, pois foi encontrada em praticamente todas as profundidades estudadas. Isoladamente, as práticas de calagem e adubação fosfatada reduzem a concentração de atrazina no solo sendo esse efeito mais significativo quando sob calagem em ambos os solos. As práticas de calagem e adubação fosfatada, isoladamente ou não, contribuiram para reduzir o potencial de mobilidade de atrazina nas colunas de solo, principalmente nas amostras de LV1.

PALAVRAS-CHAVE: LIXIVIAÇÃO; CONTAMINAÇÃO AMBIENTAL; CROMATOGRAFIA A GÁS.

\footnotetext{
* Doutora em Solos e Nutrição de Plantas, Universidade Federal de Lavras (UFLA), Campus Unibersitário Lavras, MG (e-mail: fernanda.coordenadora@gmail.com).

** Professor, Universidade Federal do Piauí (UFPI), Teresina, PI (e-mail: jnobrega@ufpi.br).

*** Professor, UFLA, Lavras, MG (e-mail: mcguerrero@ufla.br).

**** Mestranda em Ciência do Solo, UFLA, Lavras, MG (e-mail: elenalvarenga@yahoo.com.br).

***** Mestrando em Ciência do Solo, UFLA, Lavras, MG (e-mail: Igfj2002@yahoo.com.br).

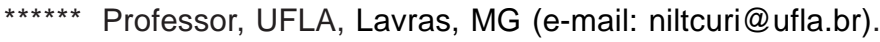




\section{INTRODUÇÃO}

Cerca de $30 \%$ da produção mundial de pesticidas corresponde aos herbicidas do grupo das triazinas (CABRAL et al., 2003), cujas principais propriedades são determinadas pelo substituinte -Cl na posição 2 do anel heterocíclico que determina o nome comercial do produto com a terminação "-azina". Outras substituições também podem ocorrer como -SCH3 ("-trina") e -OCH3 ("-tona"). No Brasil, há cerca de vinte formulações comerciais registradas com o ingrediente ativo atrazina.

As triazinas, principalmente a atrazina [2-cloro-4-etilamino-6-isopropilamino-s-triazina], são utilizadas como herbicida seletivo em doses que não excedem $3,0 \mathrm{~kg}$ de ingrediente ativo por hectare, dependendo do tipo de solo (ANDREI, 1999). São recomendadas para o controle de plantas daninhas em pré e pós-emergência nas culturas de milho, cana-de-açúcar, sorgo, cacau, banana e abacaxi, entre outras (RODRIGUES e ALMEIDA, 1998).

O uso de atrazina na Bacia do Rio das Mortes (MG) tem ocorrido principalmente na cultura do milho. Como em outras regiões do Brasil que fazem aplicação desse produto, estudos sobre a ocorrência de resíduos em solos são praticamente inexistentes, principalmente quando se leva em conta o efeito de práticas de manejo do solo e da fertilidade.

Solos tropicais altamente intemperizados, como os Latossolos que ocupam a maior parte do território brasileiro (EMBRAPA, 2006) e da Bacia do Rio das Mortes (MG) (MARQUES, CURI e LIMA et al., 2002), necessitam de práticas corretivas e de manejo da fertilidade. Tais solos apresentam, entre outras limitações, acidez elevada, baixo teor de nutrientes e de capacidade de troca catiônica, considerável saturação por alumínio trocável e capacidade substancial de retenção de fosfato (PAVAN, BINGHAM e PRATT, 1985; MIELNICZUK et al., 2003). Para essas condições edáficas, LOPES (1999) tem ressaltado a importância das práticas de calagem e adubação fosfatada visando a melhoria da fertilidade desses solos. Tais práticas promovem aumento na disponibilidade de nutrientes, redução da acidez do solo e da capacidade de adsorção de fosfato. Além dos benefícios dessas práticas na melhoria das condições químicas do solo, estudos têm mostrado que as mesmas podem influenciar o comportamento químico das moléculas de pesticidas no solo. Ao modificarem o balanço de cargas existente no processo de adsorção das moléculas de pesticidas aos constituintes orgânicos e inorgânicos do solo (DOUSSET, MOUVET e SCHIAVON, 1995; ARANTES, 2005; PLAKAS et al., 2006) alteram a taxa de degradação biológica (NAKAGAWA e ANDRÉA, 2000), ou química da molécula (ANDRÉA et al., 1997) e seu transporte para outros compartimentos do ecossistema (OLIVEIRA JÚNIOR, 1998; NÓBREGA, 2004).

No sistema solo, a atrazina pode ser adsorvida, dessorvida ou transformada parcial ou totalmente em outras moleculas (OLIVEIRA JÚNIOR, 1998; PEIXOTO et al., 2000; PARK et al., 2003; PEIXOTO et al., 2005). Na solução do solo, a molécula pode também ser absorvida e removida pelo sistema radicular das plantas e/ou outros organismos do solo. Todos esses fatores interferem na disponibilidade e, conseqüentemente, no transporte da molécula. Essa pode movimentar-se verticalmente por lixiviação para camadas subsuperficiais do perfil do solo, escoar superficialmente ("runoff') e/ou volatilizar-se. Tais circunstâncias podem ocorrer simultaneamente no solo em intensidades variáveis (FLURY, 1996; LAABS et al., 2000; PRATA, 2002; CORREIA et al., 2007) e segundo MACIEL (2002) refletem a interação do equilíbrio entre adsorção e dessorção, conteúdo de água no solo e atividade microbiana.

As rotas da molécula de atrazina nos diferentes compartimentos ambientais dependem das propriedades físicas, químicas e biológicas do solo (LAVORENTI, PRATA e REGITANO, 2003), das condições meteorológicas (PARAíBA et al., 2003), das práticas de manejo do solo e fertilidade (BOIVIN, CHERRIER e SCHIAVON, 2005; ARANTES et al., 2006; URZEDO et al., 2006), bem como das propriedades físico-químicas da molécula (JAVARONI, LANDGRAF e REZENDE, 1999; HSBD, 2006). Segundo LAVORENTI et al. (2003), apesar do incremento nas pesquisas sobre o comportamento de pesticidas em vários cenários, estudos nas condições de clima e solos brasileiros são ainda incipientes, bem como aqueles que caracterizam áreas contaminadas e as possíveis técnicas de remediação. Por isso, estudos sobre transferência de moléculas de pesticidas nos diferentes compartimentos ambientais são extremamente importantes, principalmente em áreas agrícolas cuja aplicação ocorre sob diferentes condições ambientais e práticas de manejo do solo e das culturas. 
Este estudo englobou a Bacia do Rio das Mortes (MG), utilizando solos representativos com histórico de aplicação de atrazina, tendo-se em mente a possibilidade de contaminação do solo e da água. Mais especificamente, este trabalho teve por objetivo avaliar a mobilidade do produto em colunas de lisímetro sob efeito das práticas de calagem e adubação fosfatada em Latossolos Vermelhos com teores diferenciais de óxidos de ferro.

\section{MATERIAL E MÉTODOS}

\subsection{COLETAE PREPARO DAS AMOSTRAS DE SOLO PARAESTUDO DE MOBILIDADE EM LISÍMETRO}

Amostras de Latossolo Vermelho com teores diferenciais de óxidos de ferro (mesoférrico - LV1 e hipoférrico-LV2) foram coletadas na profundidade de $0-0,20 \mathrm{~m}$ em área sob vegetação de floresta tropical subperenifólia, com relevo suave ondulado. Após a coleta, parte das amostras de solo foi seca ao ar, destorroada e peneirada em malha de $4 \mathrm{~mm}$ para enchimento das colunas no lisímetro. A outra parte foi peneirada em malha de $2 \mathrm{~mm}$ para a caracterização física, química e mineralógica. Dividiu-se esse material em quatro subamostras, sendo metade das amostras destinadas ao enchimento das colunas incubadas com calcário $\left(\mathrm{CaCO}_{3}\right)$ durante um mês. Manteve-se a umidade em torno de $60 \%$ do volume total de poros em doses equivalentes a 3,1 tha-1 para o LV1 e 4,6 $\mathrm{t} \mathrm{ha}^{-1}$ para o $\mathrm{LV} 2$, visando elevar o $\mathrm{pH}$ a 6,4. A outra subamostra foi incubada por um mês com fosfato $\left(\mathrm{KH}_{2} \mathrm{PO}_{4}\right)$ em dose equivalente a $384,61 \mathrm{mg} \mathrm{kg}^{-1}$ para o LV1 e $500 \mathrm{mg} \mathrm{kg}^{-1}$ para o LV2, que correspondem à metade da capacidade máxima de adsorção de fósforo (CMAP) determinada segundo OLSEN e WATANABE (1957). A terceira subamostra recebeu calagem e adubação fosfatada conjuntamente e a quarta não recebeu nenhum tipo de tratamento.

\subsection{CARACTERIZAÇÃO FÍSICA, QUÍMICA E MINERALÓGICA DAS AMOSTRAS DE SOLOS}

Nas amostras de solo coletadas para avaliação da mobilidade da atrazina em colunas de lisímetro, a caracterização física do solo foi efetuada mediante granulometria pelo método da pipeta (DAY, 1965), estabilidade de agregados em água (KEMPER e ROSENAU, 1986) e porcentagem de agregados maior que $2 \mathrm{~mm}$ (Tabela 1). Na caracterização química foram medidos os teores de óxidos $\left(\mathrm{Al}_{2} \mathrm{O}_{3}\right.$ e $\left.\mathrm{Fe}_{2} \mathrm{O}_{3}\right)($ Tabela 2), extraídos pelo ataque sulfúrico, segundo metodologia da EMBRAPA (1997) e a capacidade máxima de adsorção de fósforo (CMAP) (Tabela 2), determinada segundo OLSEN e WATANABE (1957). O pH em água, os cátions integrantes do complexo sortivo, o fósforo disponível e o carbono orgânico total foram determinados nas amostras de solo antes (Tabela 2) e após o período de incubação com os respectivos tratamentos (Tabela 3), segundo métodos da EMBRAPA (1997). A caracterização mineralógica foi feita por difratometria de raios-X em tubos de $\mathrm{Cu}(\lambda=0,15418 \mathrm{~nm})$ (Figura 1).

\section{TABELA 1 - ATRIBUTOS FÍSICOS DOS LATOSSOLOS VERMELHOS MESOFÉRRICO (LV1) E HIPOFÉRRICO (LV2) DA REGIÃO DA BACIA DO RIO DAS MORTES-MG, ANTES DA APLICAÇÃO DOS TRATAMENTOS}

\begin{tabular}{|c|c|c|c|c|c|c|c|c|}
\hline Solo & Areia & Silte & Argila & $\mathrm{DMG}^{1}$ & $\begin{array}{c}\text { Agr. }>2^{2} \\
m m\end{array}$ & $\mathrm{DS}^{3}$ & $D P^{4}$ & VTP $^{5}$ \\
\hline & 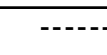 & dag kg & - - - & $\cdots$ & \% & $-\cdots \quad 9$ & $\mathrm{i}^{-3} \quad---$ & -- \% --- \\
\hline LV1 & 35 & 17 & 48 & 4,9 & 99,4 & 1,2 & 2,56 & 62,7 \\
\hline LV2 & 36 & 10 & 54 & 4,7 & 97,4 & 1,1 & 2,53 & 64,3 \\
\hline
\end{tabular}

${ }^{1}$ Diâmetro médio geométrico; ${ }^{2}$ Agregados pré-umedecidos estáveis em água com diâmetro maior que 2 mm; ${ }^{3}$ Densidade do solo; ${ }^{4}$ Densidade de partículas; ${ }^{5}$ Volume total de poros. 


\section{FIGURA 1 - DIFRATOGRAMAS DE RAIOS-X (TUBO DE CU, $\lambda=0,15418$ NM) DA FRAÇÃO ARGILA (MÉTODO DO PÓ) PARA AS AMOSTRAS DE LATOSSOLOS VERMELHOS COM TEORES DIFERENCIAIS DE ÓXIDOS DE FERRO \\ DA BACIA DO RIO DAS MORTES (MG)}

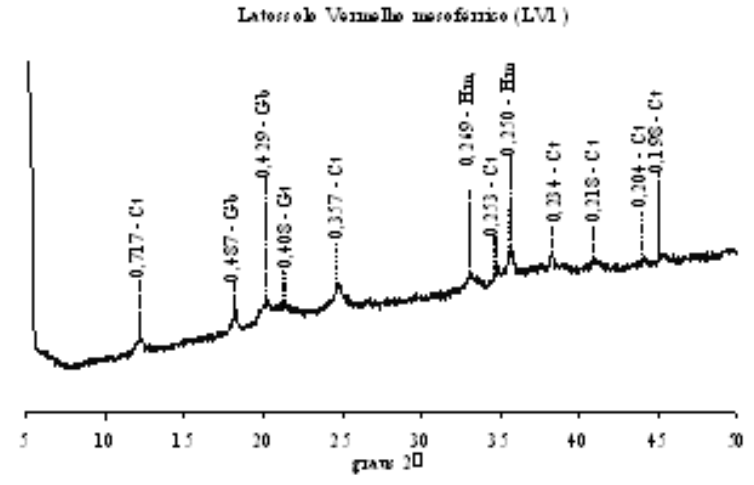

graus $2 \theta$

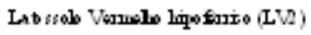

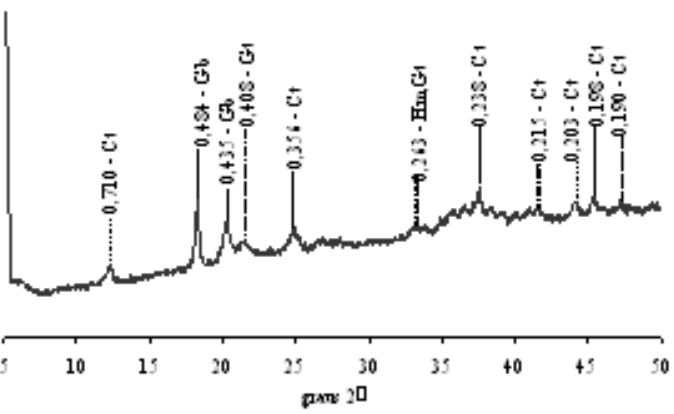

graus $2 \theta$

Números representam espaçamento D em nm. CT = Caulinita; GB = Gibbsita; GT = Goethita; HM = Hematita.

TABELA 2 - ATRIBUTOS QUÍMICOS DOS LATOSSOLOS VERMELHOS COM TEORES DIFERENCIAIS DE ÓXIDOS DE FERRO (MESOFÉRRICO - LV1; HIPOFÉRRICO - LV2), ANTES DA APLICAÇÃO DOS TRATAMENTOS

\begin{tabular}{|c|c|c|}
\hline \multirow[b]{2}{*}{ Atributo } & \multicolumn{2}{|c|}{ Solos } \\
\hline & LV1 & LV2 \\
\hline $\mathrm{pH}\left(\mathrm{H}_{2} \mathrm{O}\right)$ & 5,3 & 4,3 \\
\hline P-Mel $\left(m g d^{-3}\right)^{1}$ & 1,3 & 1,0 \\
\hline P-Rem $\left(\mathrm{mg} \mathrm{dm}^{-3}\right)^{2}$ & 24,5 & 7,6 \\
\hline $\mathrm{K}^{+}\left(\mathrm{mg} \mathrm{dm^{-3 }}\right)$ & 88,3 & 20,3 \\
\hline $\mathrm{Ca}^{2+}\left(\mathrm{cmol}_{\mathrm{c}} \mathrm{dm}^{-3}\right)$ & 2,7 & 0,4 \\
\hline $\mathrm{Mg}^{2+}\left(\mathrm{cmol}_{\mathrm{c}} \mathrm{dm}^{-3}\right)$ & 1,3 & 0,1 \\
\hline $\mathrm{SB}\left(\mathrm{cmol}_{\mathrm{c}} \mathrm{dm}^{-3}\right)^{3}$ & 4,2 & 0,6 \\
\hline $\mathrm{t}\left(\mathrm{cmol}_{\mathrm{c}} \mathrm{dm}^{-3}\right)^{4}$ & 4,5 & 1,9 \\
\hline $\mathrm{T}\left(\mathrm{cmol}_{\mathrm{e}} \mathrm{dm}^{-3}\right)^{5}$ & 9,2 & 8,6 \\
\hline$V(\%)^{6}$ & 45,0 & 7,0 \\
\hline $\mathrm{A}^{3+}\left(\mathrm{cmol}_{c} \mathrm{dm}^{-3}\right)$ & 0,3 & 1,2 \\
\hline$m(\%)^{7}$ & 7,0 & 67,0 \\
\hline COT $\left(\mathrm{g} \mathrm{kg}^{-1}\right)^{8}$ & 31,0 & 21,0 \\
\hline $\mathrm{Al}_{2} \mathrm{O}_{3}$ & 22,51 & 25,84 \\
\hline $\mathrm{Fe}_{2} \mathrm{O}_{3}(\%)$ & 15,12 & 7,72 \\
\hline $\mathrm{CMAP}^{9}\left(\mathrm{mg} \mathrm{dm}^{-3}\right)$ & 769 & 1000 \\
\hline $\mathrm{Ki}$ & 1,10 & 0,61 \\
\hline $\mathrm{Kr}$ & 0,77 & 0,50 \\
\hline
\end{tabular}

${ }^{1} \mathrm{P}$-Mel = P-Mehlich-1; ${ }^{2} \mathrm{P}$-rem = P-remanescente; ${ }^{3} \mathrm{SB}=$ soma de bases; ${ }^{4} \mathrm{t}=\mathrm{CTC}$ efetiva; ${ }^{5} \mathrm{~T}=\mathrm{CTC}$ potencial; ${ }^{6} \mathrm{~V}=$ saturação por bases; ${ }^{7} \mathrm{~m}$ = saturação por Al; ${ }^{8} \mathrm{COT}$ = carbono orgânico total; ${ }^{9} \mathrm{CMAP}$ = capacidade máxima de adsorção de fósforo. 


\section{TABELA 3 - ATRIBUTOS QUÍMICOS DOS LATOSSOLOS VERMELHOS COM TEORES DIFERENCIAIS DE ÓXIDOS DE FERRO (MESOFÉRRICO - LV1; HIPOFÉRRICO - LV2), APÓS A CALAGEM E ADUBAÇÃO FOSFATADA}

\begin{tabular}{ccccccccc}
\hline \multirow{2}{*}{ Atributo } & \multicolumn{2}{c}{ Controle } & \multicolumn{2}{c}{ Calagem } & \multicolumn{2}{c}{ Adub. fosfatada } & \multicolumn{2}{c}{ Cal. + Fosf. } \\
\cline { 2 - 9 } & LV1 & LV2 & LV1 & LV2 & LV1 & LV2 & LV1 & LV2 \\
\hline $\mathrm{pH}$ & 5,3 & 4,3 & 6,0 & 6,0 & 5,4 & 5,4 & 6,2 & 6,4 \\
P-Mel $\left(\mathrm{mg} \mathrm{dm}^{-3}\right)^{1}$ & 1,3 & 1,0 & 1,9 & 0,9 & 51,2 & 54,6 & 48,8 & 46,3 \\
P-Res $\left(\mathrm{mg} \mathrm{dm}^{-3}\right)^{2}$ & 3,3 & 1,0 & 3,2 & 2,8 & 133,3 & 155,2 & 115,3 & 168,8 \\
P-Rem $\left(\mathrm{mg} \mathrm{dm}^{-3}\right)^{3}$ & 24,5 & 7,6 & 17,8 & 12,0 & 22,4 & 16,9 & 23,3 & 20,9 \\
$\mathrm{~K}^{+}\left(\mathrm{mg} \mathrm{dm}^{-3}\right)$ & 88,3 & 20,3 & 52,0 & 31,2 & 274,7 & 135,6 & 107 & 287,3 \\
$\mathrm{Ca}^{2+}\left(\mathrm{cmol}_{\mathrm{c}} \mathrm{dm}^{-3}\right)$ & 2,7 & 0,4 & 4,5 & 3,0 & 3,3 & 0,5 & 4,4 & 2,9 \\
$\mathrm{Mg}^{2+}\left(\mathrm{cmol}_{\mathrm{c}} \mathrm{dm}^{-3}\right)$ & 1,3 & 0,1 & 1,9 & 1,4 & 1,2 & 0,2 & 2,3 & 1,5 \\
$\mathrm{SB}\left(\mathrm{cmol}_{\mathrm{c}} \mathrm{dm}^{-3}\right)^{4}$ & 4,2 & 0,6 & 6,6 & 4,5 & 5,2 & 1,1 & 6,9 & 5,1 \\
$\mathrm{t}\left(\mathrm{cmol}_{\mathrm{c}} \mathrm{dm}^{-3}\right)^{5}$ & 4,5 & 1,9 & 6,6 & 4,5 & 5,4 & 1,8 & 6,9 & 5,1 \\
$\mathrm{~T}\left(\mathrm{cmol}_{\mathrm{c}} \mathrm{dm}^{-3}\right)^{6}$ & 9,2 & 8,6 & 9,6 & 7,4 & 10,6 & 8,1 & 10,1 & 8,3 \\
${\mathrm{~V}(\%)^{7}}^{3+}\left(\mathrm{cmol}_{\mathrm{c}} \mathrm{dm}^{-3}\right)$ & 45,0 & 7,0 & 69,0 & 61,0 & 49,0 & 14,0 & 68,0 & 62,0 \\
${\mathrm{~m}(\%)^{8}}^{8}$ & 7,3 & 1,2 & 0,0 & 0,0 & 0,2 & 0,7 & 0,0 & 0,0 \\
$\mathrm{COT}\left(\mathrm{g} \mathrm{kg}^{-1}\right)^{9}$ & 31,0 & 21,0 & 31,0 & 22,0 & 31,0 & 21,0 & 31,0 & 21,0 \\
\hline
\end{tabular}

${ }^{1} \mathrm{P}-\mathrm{Mel}=\mathrm{P}$-Mehlich-1; ${ }^{2} \mathrm{P}$-Res $=\mathrm{P}$-resina; ${ }^{3} \mathrm{P}$-rem $=\mathrm{P}$-remanescente; ${ }^{4} \mathrm{SB}=$ soma de bases; ${ }^{5 \mathrm{t}}=\mathrm{CTC}$ efetiva; ${ }^{6} \mathrm{~T}=\mathrm{CTC}$ potencial; ${ }^{7} \mathrm{~V}$ = saturação por bases; ${ }^{8} \mathrm{~m}$ = saturação por $\mathrm{Al} ;{ }^{9} \mathrm{COT}$ = carbono orgânico total.

\subsection{MONTAGEM DAS COLUNAS DE SOLO NOS LISÍMETROS E ESTABILIZAÇÃO COM SOLUÇÃO DE $\mathrm{CACl}_{2}\left(0,01 \mathrm{MOL} \mathrm{L}^{-1}\right)$}

Após o período de incubação, as amostras de solo (aproximadamente $30 \mathrm{~kg}$ ) foram colocadas em colunas com dimensões de $0,30 \times 0,30 \times 0,35 \mathrm{~m}$. Os tratamentos foram dispostos em delineamento experimental inteiramente casualizado, esquema fatorial $2 \times 2$, sendo testados os seguintes fatores: solos em duas condições de fósforo (com e sem fosfato) e duas condições de calagem (com e sem calagem), com três repetições, totalizando 12 parcelas experimentais para cada solo.

Antes da aplicação do ingrediente ativo foi necessário efetuar o equilíbrio da força iônica nos diferentes tratamentos. Para isso, nas colunas dos lisímetros foram realizadas lixiviações com solução de $\mathrm{CaCl}_{2}\left(0,01 \mathrm{~mol} \mathrm{~L}^{-1}\right)$ e a força iônica do percolado avaliada até que não mais existisse diferença entre os tratamentos. Estimou-se a força iônica a partir da condutividade elétrica, utilizando a fórmula $\mu=0,013 E C$ em que $\mu$ é a força iônica baseada na concentração expressa em mol L-1 e $E C$ corresponde à condutividade elétrica expressa em $\mathrm{m} \Omega \mathrm{cm}^{-2}$, a temperatura de $25^{\circ} \mathrm{C}$ (LINDSAY, 1979).

\subsection{APLICAÇÃO DO HERBICIDA ATRAZINAE SIMULAÇÃO DE CHUVAS NAS COLUNAS DO LISÍMETRO}

A solução de atrazina foi aplicada com auxílio de pipeta $(5 \mathrm{~mL})$, gota a gota, em dose única, correspondendo a $6 \mathrm{~L} \mathrm{~h}^{-1}$ do produto comercial Gesaprim $500^{\circledR}$ (ANDREI, 1999), ou à aproximadamente $27 \mathrm{mg}$ de ingrediente ativo por coluna de solo, imaginando um quadrado com margem de 2,0 cm das 
bordas das células do lisímetro e, em ziguezague, em sua área interna. Após 12 horas da aplicação do produto efetuou-se a primeira simulação de chuva, usando recipientes plásticos com fundo perfurado, à semelhança do trabalho de NÓBREGA (2004). A quantidade de chuva simulada foi de $18,75 \mathrm{~mm}$, correspondente a aplicação de 1,70 L de água deionizada em cada coluna. Esse procedimento ocorreu a cada três dias, durante 1 mês, totalizando a aplicação de $187,5 \mathrm{~mm}$ de água que corresponde a média de chuvas nos meses de outubro e novembro, principal época do plantio de milho na região da Bacia do Rio das Mortes, MG (VASCONCELOS, 2007).

Tanto no momento da aplicação do produto como em todo o período de condução do experimento, o solo foi mantido com teor de água na capacidade de campo (FREIRE et al., 1980). Para isso controlou-se a perda de água nas colunas de solo por evaporação pela pesagem diária de 1 vaso com área superficial similar a das colunas de solo, sendo a quantidade de água perdida por evaporação restituída à coluna no dia seguinte.

\subsection{COLETA DO PERCOLADO E RETIRADA DO SOLO DAS COLUNAS DO LISÍMETRO}

O material percolado em cada coluna foi coletado em recipientes cilíndricos $(2,5 \mathrm{~L})$ de aço inox, após cada simulação de chuva. Mediu-se o volume total percolado com auxílio de proveta de $2 \mathrm{~L}$, sendo uma alíquota de $50 \mathrm{~mL}$ conservada em frascos âmbar a $-18^{\circ} \mathrm{C}$ para posterior análise cromatográfica.

Ao final do período de lixiviação, com auxílio de bandejas plásticas, o solo contido em cada coluna foi retirado por camadas de $0-5 ; 5-10 ; 10-15 ; 15-20 ; 20-25$ e $25-30 \mathrm{~cm}$ e homogeneizado. Uma fração de cada camada foi armazenada em sacos plásticos e conservada a $-18^{\circ} \mathrm{C}$ para posterior avaliação da concentração de atrazina (VASCONCELOS, 2007).

\subsection{EXTRAÇÃO E QUANTIFICAÇÃO DA ATRAZINANAS AMOSTRAS DE SOLO E ÁGUA PERCOLADA}

Para a extração da atrazina nas amostras de solo, coletadas em cada profundidade das colunas do lisímetro, utilizaram-se $100 \mathrm{~g}$ de solo para $100 \mathrm{~mL}$ da solução extratora (metanol p.a.), deixando a mistura por 30 minutos em mesa de agitação. Após o período de repouso para decantação da suspensão (aproximadamente 20 minutos), o sobrenadante foi recolhido e depositado em frasco plástico com tampa. Repetiu-se tal procedimento por mais duas vezes. Posteriormente, o sobrenadante foi centrifugado a $3.000 \mathrm{rpm}$ por 10 minutos e transferido para balão de fundo redondo. Evaporou-se o solvente em evaporador rotativo sob vácuo a $40^{\circ} \mathrm{C}$ até a obtenção de alíquota de aproximadamente $5 \mathrm{~mL}$.

Filtrou-se o extrato em membrana de $20 \mu \mathrm{m}$ e $1 \mu \mathrm{L}$ foi injetado durante 1 minuto (injeção do tipo splitless) em cromatógrafo a gás (CG), Hewlett Packard, modelo 6890, equipado com detector de nitrogênio e fósforo (NPD) e coluna HP-5 (30 m x $320 \mu \mathrm{m} \times 0,25 \mu \mathrm{m}$ com 5\% de fenil-metil-siloxano). Os parâmetros para a operação do aparelho foram: temperatura do injetor $280^{\circ} \mathrm{C}$; temperatura do detector $300^{\circ} \mathrm{C}$; temperatura inicial do forno $120^{\circ} \mathrm{C}$ com duração de 1 minuto; rampa de aquecimento $50^{\circ} \mathrm{C} \mathrm{min}{ }^{-1}$ até $250^{\circ} \mathrm{C}$, permanecendo 1 minuto e 40 segundos. Usou-se $\mathrm{N}_{2}$ como gás de arraste e fluxo aproximado de 1,0 a 1,5 mL min-1. Efetuou-se a padronização mediante curva analítica com padrão externo. Nessas condições, o tempo de retenção da atrazina foi de 4 minutos e 42 segundos.

Determinou-se, quantitativamente, a concentração de atrazina nas amostras de solo por comparação das alturas dos picos das amostras com os da curva de calibração (ANALYTICAL METHODS COMMITTED, 1987), obtida pela injeção $1 \mu \mathrm{L}$ das soluções-padrão de Gesaprim 500 ${ }^{\circledR}$, contendo concentrações que variavam de 31,25 a $1.000 \mu \mathrm{g} \mathrm{kg}^{-1}$.

Para avaliação da concentração de atrazina na água percolada efetuou-se somente a filtração do percolado em membrana de $20 \mu \mathrm{m}$ e $1 \mu \mathrm{L}$ foi injetado no cromatógrafo nas mesmas condições descritas anteriormente para a análise de atrazina nas amostras de solo. 


\subsection{ANÁLISE ESTATÍSTICA}

Para cada um dos Latossolos Vermelhos estudados (LV1 e LV2), os valores médios da concentração de atrazina foram calculados para cada camada $(0-5 ; 5-10 ; 10-15 ; 15-20 ; 20-25$; e $25-30 \mathrm{~cm}$ ) e tratamento (presença e ausência da calagem e/ou adubação fosfatada) com os respectivos desvios-padrão (FERREIRA, 2000).

\section{RESULTADOS E DISCUSSÃO}

\subsection{AVALIAÇÃO DA MOBILIDADE DA ATRAZINA EM COLUNAS DE LISÍMETRO E ÁGUA PERCOLADA}

O efeito das práticas de calagem e adubação fosfatada sobre a concentração de atrazina em profundidade nas colunas dos lisímetros encontra-se na Tabela 4.

A molécula de atrazina apresentou, em ambos os solos, potencial de mobilidade em profundidade na maioria das amostras. Tal fato permite classificá-la como molécula de média mobilidade no solo (RODRIGUES e ALMEIDA, 1998; LAABS et al., 2000; PRATA, 2002), pois atingiu a profundidade média de $25 \mathrm{~cm}$ no LV2, independente do tratamento aplicado e $20 \mathrm{~cm}$ no LV1, quando submetida à prática de adubação fosfatada.

A mobilidade da atrazina em profundidade no perfil do solo também foi verificada por BARRIUSO et al. (1992); LAABS et al. (2000); CERDEIRA et al. (2005); CORREIA e LANGENBACH (2006). LAABS et al. (2000) ao estudarem a lixiviação e degradação de pesticidas em áreas sob cultivo de milho e feijão verificaram moderada lixiviação da atrazina em Latossolo Roxo distrófico. Segundo esses autores, $0,46 \%$ da massa de atrazina foi encontrada a $15 \mathrm{~cm}$ de profundidade no lisímetro após 28 dias da aplicação do produto. Efeito semelhante foi relatado em solos oxídicos por BARRIUSO et al. (1992). CERDEIRA et al. (2005) constataram em colunas contendo amostras de Latossolo Vermelho eutroférrico a movimentação da atrazina até a profundidade máxima de $20 \mathrm{~cm}$, enquanto CORREIA e LANGENBACH (2006) verificaram que aproximadamente $15 \%$ da massa de atrazina aplicada foi distribuída de forma gradativa e decrescente até a profundidade de $25 \mathrm{~cm}$.

Segundo HUANG e FRINK (1989), a movimentação do pesticida até determinada profundidade no perfil está correlacionada com a distribuição do teor de carbono orgânico (BARRIUSO et al., 1992) e o tamanho das partículas constituintes do solo, principalmente, o teor de argila.

Ocorreram valores semelhantes de carbono orgânico total nas diferentes camadas do LV1 e LV2 (Tabela 5), embora com diferenças entre os solos e valores mais elevados no LV1. Comportamento análogo foi verificado para a porcentagem de argila nas diferentes profundidades de ambos os solos. A menor concentração de atrazina verificada nas amostras do LV1, exceto na camada $0-5 \mathrm{~cm}$ do tratamento controle, pode ser explicada pela ocorrência de teor mais elevado de carbono orgânico total. Provavelmente, isso contribuiu para a maior retenção, além de outros processos de dissipação da molécula (degradação, formação de resíduo-ligado, etc.) que reduzem a mobilidade da molécula ao longo do perfil do solo.

A formação de resíduo-ligado no LV1, provavelmente, ocorreu com a matéria orgânica (Tabelas 3 e 4) e no LV2, com a goethita (Figura 1). Maior adsorção de atrazina tem sido registrada nos óxidos de ferro (MOREAU e MOUVET, 1998) e nos ácidos húmicos (MATIN NETO et al., 1994). Em Latossolos, o maior teor de óxidos de ferro e de alumínio tende a neutralizar as cargas negativas da matéria orgânica, provavelmente reduzindo sua solvatação pelas moléculas de água e possibilitando maior interação com o produto (URZEDO et al., 2006).

Ao observar o efeito das práticas de calagem e adubação fosfatada, isoladamente ou em conjunto na concentração de atrazina nas diferentes camadas em profundidade nas colunas (Tabela 4), verifica-se que as mesmas contribuem para redução da concentração de atrazina, quando se comparam as concentrações desses tratamentos com as amostras controle de ambos os solos. 


\section{TABELA 4 - CONCENTRAÇÃO DE ATRAZINA EM AMOSTRAS DE LATOSSOLO VERMELHO MESOFÉRRICO (LV1) E LATOSSOLO VERMELHO HIPOFÉRRICO (LV2) SOB EFEITO DA CALAGEM E ADUBAÇÃO FOSFATADA}

\begin{tabular}{|c|c|c|c|}
\hline \multirow[b]{2}{*}{ Tratamento } & \multirow[b]{2}{*}{ Profundidade } & \multicolumn{2}{|c|}{ Latossolos } \\
\hline & & LV1 & LV2 \\
\hline \multirow{7}{*}{ Controle } & --- cm --- & \multicolumn{2}{|c|}{-------Concentração $\left(\mu \mathrm{g} \mathrm{kg}^{-1}\right)$} \\
\hline & $0-5$ & $43,61 \pm 6,60$ & $36,50 \pm 5,73$ \\
\hline & $5-10$ & $10,78 \pm 3,32$ & $18,15 \pm 6,33$ \\
\hline & $10-15$ & $1,91 \pm 1,27$ & $12,76 \pm 4,38$ \\
\hline & $15-20$ & nd & $4,32 \pm 3,05$ \\
\hline & $20-25$ & nd & $<1$ \\
\hline & $25-30$ & nd & nd \\
\hline \multirow[t]{6}{*}{ Calagem } & $0-5$ & $7,30 \pm 1,54$ & $7,30 \pm 3,61$ \\
\hline & $5-10$ & $2,71 \pm 0,88$ & $8,59 \pm 3,75$ \\
\hline & $10-15$ & $<1$ & $5,42 \pm 1,47$ \\
\hline & $15-20$ & nd & $2,31 \pm 0,99$ \\
\hline & $20-25$ & nd & $2,66 \pm 2,33$ \\
\hline & $25-30$ & nd & nd \\
\hline \multirow{6}{*}{$\begin{array}{l}\text { Adubação } \\
\text { fosfatada }\end{array}$} & $0-5$ & $15,55 \pm 1,76$ & $22,94 \pm 10,28$ \\
\hline & $5-10$ & $3,93 \pm 0,92$ & $16,21 \pm 0,73$ \\
\hline & $10-15$ & $1,40 \pm 0,60$ & $13,37 \pm 3,69$ \\
\hline & $15-20$ & $<1$ & $2,12 \pm 1,39$ \\
\hline & $20-25$ & nd & $<1$ \\
\hline & $25-30$ & nd & nd \\
\hline \multirow{6}{*}{$\begin{array}{l}\text { Calagem + } \\
\text { Adubação } \\
\text { fosfatada }\end{array}$} & $0-5$ & $<1$ & $4,97 \pm 3,08$ \\
\hline & $5-10$ & nd & $3,45 \pm 0,52$ \\
\hline & $10-15$ & nd & $1,56 \pm 0,36$ \\
\hline & $15-20$ & nd & $1,36 \pm 0,82$ \\
\hline & $20-25$ & nd & $<1$ \\
\hline & $25-30$ & $\mathrm{Nd}$ & $\mathrm{Nd}$ \\
\hline
\end{tabular}

nd $=$ não-detectado.

Nos solos sob calagem (Tabela 4), a concentração de atrazina na camada de $0-5 \mathrm{~cm}$ foi aproximadamente seis vezes menor nas amostras do LV1 e cinco vezes menor nas do LV2 em relação às amostras controle. Na camada de $5-10 \mathrm{~cm}$, a redução foi de 4,0 e 2,1 vezes, respectivamente. Redução mais significativa na concentração de atrazina nas amostras do LV1 explica sua menor mobilidade nesse tratamento $(15 \mathrm{~cm})$ em relação à amostra do LV2 $(25 \mathrm{~cm})$.

O efeito isolado da calagem na redução da concentração de atrazina, principalmente, no LV1 deve-se à maior dissipação da molécula na forma de resíduo ligado à matéria orgânica (BOLLAG e LIU, 1990; NAKAGAWA e ANDREA, 1997; NAKAGAWA e ANDREA, 2000; PRATA, 2002; LAVORENTI, PRATA e REGITANO, 2003; PEIXOTO et al., 2005; CORREIA e LANGENBACH, 2006) e/ou aumento na concentração de cálcio, que reduz a interação da molécula do pesticida à matéria orgânica (PLAKAS 
et al., 2006). Outra razão seria a maior taxa de degradação da molécula pelos microrganismos do solo, explicada pela melhoria nas suas condições de fertilidade (BOLLAG e LIU, 1990; PEIXOTO et al., 2000; LAVORENTI et al., 2003; TOMITA et al., 2004) (Tabela 3).

PLAKAS et al. (2006) ao estudarem a eficiência de nanomembranas na retenção de algumas moléculas, dentre as quais a atrazina, verificaram que a presença de íons cálcio em solução diminui a retenção do herbicida. Os mesmos interferem nas interações da atrazina com os ácidos húmicos e fúlvicos, componentes da matéria orgânica, conforme já citado por DEVITT e WIESNER (1998). Melhoria das condições de fertilidade na redução da concentração de pesticidas em solos também foi verificada por LOPES (1983) ao relatar a importância da calagem para a menor adsorção de fosfato na superfície das partículas. Desta forma, a diminuição dos sítios de retenção com a elevação do $\mathrm{pH}$ propicia redução do triadimenol nas amostras dos solos dos cerrados. Resultados semelhantes foram obtidos por NÓBREGA (2004) em estudo da retenção do triadimenol em Latossolo Vermelho distrófico, submetidos à calagem e à adubação fosfatada. Menor retenção da molécula nesses solos para a maior dose aplicada foi verificada, independente do manejo adotado.

Nos solos sob adubação fosfatada (Tabela 5), a concentração de atrazina ao longo das colunas também foi reduzida em relação às amostras controle em ambos os solos, principalmente no LV1 $(2,80$ e 2,74 vezes, respectivamente nas camadas de $0-5$ e $5-10 \mathrm{~cm})$. No entanto, a redução na concentração de atrazina mostrou-se bem inferior nas amostras com calcário. Tal fato explica a maior mobilidade da molécula nesse tratamento que atingiu a profundidade de $20 \mathrm{~cm}$ no LV1 e $25 \mathrm{~cm}$ no LV2.

\section{TABELA 5 - ATRIBUTOS QUÍMICOS DO LATOSSOLO VERMELHO MESOFÉRRICO (LV1) E LATOSSOLO VERMELHO HIPOFÉRRICO (LV2) SOB EFEITO DA CALAGEM E ADUBAÇÃO FOSFATADA}

\begin{tabular}{|c|c|c|c|c|c|c|c|c|c|}
\hline \multirow[b]{2}{*}{ Tratamento } & \multirow[b]{2}{*}{ Profundidade } & \multicolumn{8}{|c|}{ Latossolos } \\
\hline & & LV1 & LV2 & LV1 & LV2 & LV1 & LV2 & LV1 & LV2 \\
\hline \multirow{7}{*}{ Controle } & 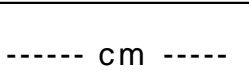 & \multicolumn{2}{|c|}{$\mathrm{pH} \mathrm{H}{ }_{2} \mathrm{O}$} & \multicolumn{2}{|c|}{$\begin{array}{l}\text { P Mehlich } \\
--\mathrm{mg} \mathrm{dm}^{-3}\end{array}$} & \multicolumn{2}{|c|}{$\begin{array}{c}P \text { resina } \\
\text { m g d } m^{-3} \ldots\end{array}$} & $\mathrm{COT}$ & $\begin{array}{l}\mathrm{T} \\
\mathrm{kg}^{-1}-\end{array}$ \\
\hline & $0-5$ & 3,6 & 4,7 & 1,4 & 1,2 & 4,5 & 3,3 & 27,3 & 13,9 \\
\hline & $5-10$ & 5,4 & 4,5 & 1,3 & 1,1 & 4,2 & 3,0 & 27,3 & 13,3 \\
\hline & $10-15$ & 5,3 & 4,4 & 1,3 & 1,1 & 4,5 & 2,9 & 27,3 & 15,1 \\
\hline & $15-20$ & 5,2 & 4,3 & 1,4 & 1,2 & 5,1 & 3,0 & 26,7 & 14,5 \\
\hline & $20-25$ & 5,2 & 4,2 & 1,4 & 1,3 & 5,3 & 3,3 & 30,7 & 15,7 \\
\hline & $25-30$ & 5,1 & 4,2 & 1,5 & 1,3 & 4,9 & 3,8 & 31,3 & 18,0 \\
\hline Calagem & $0-5$ & 6,2 & 6,1 & 1,4 & 1,2 & 3,9 & 5,3 & 27,8 & 15,7 \\
\hline & $5-10$ & 6,1 & 6,1 & 1,5 & 1,1 & 4,4 & 5,4 & 26,7 & 18,0 \\
\hline & $10-15$ & 6,1 & 6,1 & 1,3 & 1,1 & 4,2 & 5,0 & 27,3 & 18,0 \\
\hline & $15-20$ & 6,2 & 6,0 & 1,4 & 1,1 & 3,9 & 4,7 & 27,3 & 18,0 \\
\hline & $20-25$ & 6,1 & 6,0 & 1,5 & 1,1 & 4,8 & 4,9 & 30,2 & 20,3 \\
\hline & $25-30$ & 6,1 & 5,9 & 1,4 & 1,2 & 4,5 & 4,9 & 29,6 & 19,1 \\
\hline A dubação & $0-5$ & 5,8 & 4,9 & 22,8 & 36,1 & 61,0 & 106,9 & 30,2 & 13,9 \\
\hline fosfatada & $5-10$ & 5,7 & 5,1 & 42,2 & 59,7 & 103,5 & 140,8 & 26,7 & 13,9 \\
\hline & $10-15$ & 5,6 & 5,2 & 61,4 & 67,3 & 133,4 & 164,9 & 26,7 & 14,5 \\
\hline & $15-20$ & 5,5 & 5,3 & 71,6 & 81,4 & 140,5 & 186,1 & 29,6 & 17,4 \\
\hline & $20-25$ & 5,5 & 5,5 & 83,1 & 81,1 & 157,0 & 180,7 & 31,3 & 16,8 \\
\hline & $25-30$ & 5,4 & 5,3 & 50,0 & 77,6 & 150,6 & 179,6 & 31,3 & 15,7 \\
\hline Calagem + & $0-5$ & 6,4 & 6,2 & 25,7 & 29,2 & 69,3 & 149,7 & 29,0 & 18,6 \\
\hline Adubação & $5-10$ & 6,2 & 6,2 & 45,3 & 53,8 & 113,5 & 168,6 & 31,9 & 17,4 \\
\hline fosfatada & $10-15$ & 6,3 & 6,2 & 66,9 & 60,1 & 160,6 & 180,7 & 29,0 & 19,7 \\
\hline & $15-20$ & 6,2 & 6,3 & 67,6 & 70,2 & 158,6 & 185,9 & 31,3 & 20,3 \\
\hline & $20-25$ & 6,2 & 6,3 & 65,3 & 73,0 & 147,2 & 191,0 & 30,7 & 18,6 \\
\hline & $25-30$ & 6,1 & 6,3 & 72,8 & 70,7 & 157,0 & 187,5 & 30,2 & 18,0 \\
\hline
\end{tabular}


A adubação fosfatada altera o balanço de cargas no solo, pois eleva o $\mathrm{pH}$. Isto possibilita maior liberação de $\mathrm{OH}^{-}$para a solução do solo, explicada pela ligação covalente (troca de ligantes) entre os óxidos de ferro e alumínio e os íons fosfato. Tal fato foi observado por LIMA (2004) ao estudar Latossolos Vermelhos distróficos sob adubação fosfatada. A competição do íon fosfato com a matéria orgânica pelas cargas positivas dos óxidos de ferro e alumínio permite maior solvatação da matéria orgânica pela água devido ao aumento da disponibilidade dos sítios negativos, o que reduz a sua interação com o pesticida. URZEDO et al. (2004) também constataram fato semelhante ao estudarem o comportamento do tiametoxam em Latossolo Vermelho Amarelo distrófico e Latossolo Vermelho distrófico. Além disso, PRATA (2002) verificou que o glifosato forma ligação covalente com os sesquióxidos metálicos do solo de forma semelhante aos fosfatos inorgânicos. Constatou competição entre o glifosato e o fósforo quando os níveis desse no solo são inferiores a $1000 \mathrm{mg} \mathrm{dm}^{-3} \mathrm{em}^{\text {Nitossolo }}$ Vermelho eutroférrico e Latossolo Amarelo ácrico, permanecendo a molécula na forma de resíduo ligado.

Para as amostras sob calagem mais adubação fosfatada foi verificada redução na concentração de atrazina, tanto em relação às amostras controle quanto em relação aos demais tratamentos. Essa redução mostrou-se mais significativa na amostra do LV1, sendo detectada somente em concentração de atrazina inferior a $1 \mu \mathrm{g} \mathrm{kg}^{-1}$ na camada $0-5 \mathrm{~cm}$. A molécula atingiu a profundidade de $25 \mathrm{~cm}$ no LV2, embora em níveis inferiores ao tratamento sob calagem e adubação fosfatada isoladamente. A redução na concentração de atrazina nesse tratamento se deve, provavelmente, ao efeito dessas práticas na sua dissipação por processos de transformação da molécula e/ou formação de resíduoligado (ANDRÉA et al., 1997; PEIXOTO et al., 2000; PEIXOTO et al., 2005). Nos estudos realizados por LIMA (2004) não foram constatadas alterações significativas para atrazina em função da calagem e da adubação fosfatada nos solos estudados, mas ocoreu maior sorção em Latossolo Vermelho distrófico que em Latossolo Vermelho-Amarelo distrófico. Assim, o efeito das práticas de calagem e adubação fosfatada realizadas em conjunto é mais significativo para a redução da concentração de atrazina nas camadas que isoladamente.

No presente estudo não foi verificada mobilidade de atrazina além dos $25 \mathrm{~cm}$ de profundidade nas colunas, fato que evidencia a elevada capacidade de sorção e/ou dissipação da molécula nas camadas mais superficiais de ambos os solos. Além disso, não foi possível detectar a presença da mesma na água percolada. Presença de atrazina na água percolada tem sido verificada por diferentes pesquisadores (CORREIA, 2000; LAABS et al., 2000; LANGENBACH, SCHROLL e PAIM, 2000; LAABS et al., 2002; PRATA, 2002; AMARAL, 2004; YING et al., 2005). DOUSSET, MOUVET e SCHIAVON, (1995) encontraram resíduos de s-triazinas em lisímetros após 10 meses da aplicação e LAABS et al. (2000) no lixiviado de lisímetro com $35 \mathrm{~cm}$ de profundidade, 28 dias após sua aplicação.

MATOS e SILVA (1999) constataram que quanto maior a adsorção da molécula de pesticida, menor será sua mobilidade no perfil do solo. Assim, moléculas que apresentam coeficientes de adsorção mais elevados tendem a ficar retidas na camada superficial e por isso não se deslocam por lixiviação em direção às camadas mais profundas do solo. No entanto, nas moléculas (mais móveis) com valores dos coeficientes de adsorção menores, a intensidade do processo de lixiviação é influenciada por outros fatores. Tem-se, como exemplo, o tempo de meia-vida da molécula que determinará a maior ou menor presença da mesma em solução e, com isso, sua maior ou menor intensidade de lixiviação.

Estudos conduzidos por LAABS et al. (2000) mostraram que o tempo de dissipação da molécula de atrazina foi de 6,3 e 12,6 dias, respectivamente para 50 e $75 \%$ da massa do produto aplicada nas camadas superficiais de solos oxídicos. Segundo GUERREIRO et al. (2006), a degradação da atrazina segue o modelo de cinética de primeira ordem com tempo de meia-vida de aproximadamente 16 dias em Latossolo Vermelho distrófico típico o que também pode justificar a não-detecção de atrazina na água percolada. Outros fatores que explicam a ausência de resíduos de atrazina na água percolada são as práticas de manejo que contribuem para a melhoria das condições de acidez e elevação dos teores de nutrientes (Tabelas 3,4 , e 5), gerando condições menos restritivas para a microbiota do solo e, desse modo, aumentando a taxa de degradação da molécula de atrazina. 
Apesar da ausência de atrazina na água percolada, a sua distribuição em profundidade nas colunas (mesmo decrescente) mostra a capacidade de movimentação do produto na coluna de solo e, conseqüentemente, o possível risco de contaminação de águas subsuperficiais (MATOS e SILVA, 1999; RODRIGUES, 2001). Esse risco decorre do fato que sob condições de campo não há apenas uma aplicação e o volume de água percolada no perfil é significativamente superior ao usado neste estudo. Com isso, pesquisas devem ser realizadas sob essas condições objetivando verificar o maior ou menor potencial de lixiviação da atrazina.

\section{CONCLUSÃo}

Verificou-se no estudo em lisímetros que a atrazina apresenta potencial de mobilidade no perfil de ambos os solos, principalmente nas amostras de Latossolo Vermelho hipoférrico.

As práticas de calagem e adubação fosfatada, isoladamente e/ou em conjunto, reduzem o potencial de mobilidade da molécula de atrazina, em ambos os solos, devido ao aumento que promovem na dissipação da mesma.

\section{ABSTRACT}

\section{MOBILITY OF THE ATRAZINE HERBICIDE IN SAMPLES OF RED LATOSOLS UNDER LIMING AND PHOSPHATE FERTILIZATION}

The objective of this study was to evaluate the effect of liming and phosphate fertilization practices, separately or in set, on the atrazine leaching in Red Latosols samples with distinct iron oxide purports (in lysimeters), under laboratory conditions. The treatments had been ruled in totally randomized experimental design, factorial design $2 \times 2$, being the following factors tested: soils in two conditions of phosphorus (with and without phosphate), and two conditions of liming (with and without liming), with three repetitions, totalizing 12 experimental parcels for each soil. Atrazine was applied in dose corresponding to $6 \mathrm{~L}$ per hectar of Gesaprim $500^{\circledR}$ and had been simulated rains of $18.75 \mathrm{~mm}$ to each three days during the period of 30 days. The solid sampled material was prepared for extration of atrazine and quantified by gas chromatograph, equipped with detector of nitrogen and phosphorus (GC-NPD). The results showed that, independently of soils and liming and phosphate fertilization practices, atrazine presents mobility, because it was found in practically all the studied depths. Separately, liming and phosphate fertilization practices reduce the atrazine concentration in the ground, being this effect more significant when under liming in both soils. The liming and phosphate fertilization effects, separately or not, contributed to reduce the potential of mobility in the ground columns, mainly in the LV1 samples.

\section{KEY-WORDS: LEACHING; ENVIRONMENTAL CONTAMINATION; GAS CHROMATOGRAPHY.}

\section{REFERÊNCIAS}

1 AGROFIT. Sistemas de Agrotóxicos Fitossanitários. Ingrediente ativo - atrazina. Disponível em: http:// extranet.agricultura.gov.br/agrofit_cons/principal_agrofit_cons. Acesso em: 16 ago. 2005.

2 AMARAL, L. C. S. Sorção e mobilidade de atrazina em Latossolos da região de Lavras. Lavras, 2004.158 p. Tese (Doutorado em Agronomia - Solos e Nutrição de Plantas)-Universidade Federal de Lavras.

3 ANALYTICAL METHODS COMMITTEE. Recommendations for the definition, estimation and use of detection limit. The Analyst, n.112, p.199-204, 1987.

4 ANDREA, M. M.; MATALLO, M. B.; TOMITA, R. Y.; LUCHINI, L. C. Effect of temperature on dissipation of $\left[{ }^{14} \mathrm{C}\right]$-atrazine in a Brazilian soil. Pesquisa Agropecuária Brasileira, Brasília, v.32, n.1, p.95-100, 1997.

5 ANDREI, E. Compêndio de defensivos agrícolas: guia prático de produtos fitossanitários para uso agrícola. 6. ed. São Paulo: Organização Andrei Editora, 1999. 672 p.

6 ARANTES, S. A. do C. M. Sorção de atrazina em solos da bacia do Rio das Mortes e seu movimento em Latossolo Vermelho distroférrico sob plantio direto e convencional. Lavras, 2005. 78 p. Dissertação (Mestrado em Agronomia - Solos e Nutrição de Plantas), Universidade Federal de Lavras. 
7 ARANTES, S. A. do C. M.; LIMA, J. M. de; NÓBREGA, J. C. A.; GUILHERME, L. R. G.; JULIÃO, L. G. F.; JESUS, E. A. de. Sorção de atrazina em solos representativos da sub-bacia do Rio das Mortes-MG. Pesticidas: revista de ecotoxicologia e meio ambiente, Curitiba, v.16, p.101-110, 2006.

8 BARRIUSO, E.; FELLER, C.; CALVET, R.; CERRI, C. Sorption of atrazine, terbutryn and 2,4-D herbicides in two Brazilian Oxisols. Geoderma, v.53, n.1/2, p.155-167, 1992.

9 BOLLAG, J. M.; LIU, S. Y. Biological transformation processes of pesticides. In: CHENG, H. H. (Ed.) Pesticides in the soil environment: processes, impacts and modeling. Madison, WI: Soil Science Society of America, 1990. p.169-211.

10 BOIVIN, A.; CHERRIER, R.; SCHIAVON, M. A comparison of five pesticides adsorption and desorption process in thirteen contrasting field soils. Chemosphere, v.61, p.668-676, 2005.

11 CABRAL, M. F.; SOUZA, D.; ALVES, C. R.; MACHADO, S. A. S. Estudo do comportamento eletroquímico do herbicida ametrina utilizando a técnica de voltametria de onda quadrada. Eclética Química, v.28, n.2, p.41-47, 2003.

12 CERDEIRA, A. L.; SOUZA, M. D. de; PESSOA, M. C. P. Y.; QUEIROZ, S. C. N. de; FERRACINI, V. L.; LANCHONTE, V. L.; QUEIROZ, R. H. C.; BOLONHEZI, D.; FARIANI NETO, C. Efeito de sistemas de cultivo com cana-de-açúcar sobre a lixiviação dos herbicidas do grupo das triazinas em região de recarga do Aqüífero Guarani. Jaguariúna: EMBRAPA Meio Ambiente, 2005. 25 p. (Boletim de Pesquisa e Desenvolvimento, 32).

13 CORREIA, F. V. Distribuição e degradação do herbicida atrazina em solo Podzólico Vermelho-Amarelo sob condições de clima tropical úmido. Lavras, 2000. 83 p. Dissertação (Mestrado em Agronomia - Solos e Nutrição de Plantas), Universidade Federal de Lavras.

14 CORREIA, F. V.; LANGENBACH, T. Dinâmica da distribuição e degradação de atrazina em Argissolo VermelhoAmarelo sob condições de clima tropical úmido. Revista Brasileira de Ciência do Solo, v.30, p.183-192, 2006.

15 CORREIA, F. V.; MACRAE, A.; GUILHERME, L. R. G.; LANGENBACH, T. Atrazine sorption and fate in a Ultisol from humid tropical Brazil. Chemosphere, v.67, p.847-854, 2007.

16 DAY, P. R. Particle fractionation and particle-size analyses. In: BLACK, C. A.. (Ed.) Methods of soil analysis. Madison: American Society of Agronomy, 1965. v.1, p.545-566.

17 DEVITT, E. C.; WIESNER, M. R. Dialysis investigations of atrazine-organic matter interactions and the role of a divalent metal. Environmental Science Technology, v.32, p.232-237, 1998.

18 DOUSSET, S.; MOUVET, C.; SCHIAVON, M. Leaching of atrazine and some of its metabolites in undisturbed field lysimeters of three soil types. Chemosphere, v.30, n. 3, p.511-524, 1995.

19 EMBRAPA. Empresa Brasileira de Pesquisa Agropecuária. Centro Nacional de Pesquisa de Solos. Manual de métodos de análises de solo. 2.ed. Rio de Janeiro, 1997. 212 p.

20 EMBRAPA. Empresa Brasileira de Pesquisa Agropecuária. Centro Nacional de Pesquisa de Solos. Sistema brasileiro de classificação de solos. 2.ed. Rio de Janeiro, 2006. 306 p.

21 FERREIRA, D. F. Análises estatísticas por meio do SISVAR para Windows versão 4.0. In: REUNIÃO ANUAL DA REGIÃO BRASILEIRA DA SOCIEDADE INTERNACIONAL DE BIOMETRIA, 45., 2000, São Carlos, SP. Anais... São Carlos: UFSCar, 2000. p. 235.

22 FLURY, M. Experimental evidence of transport of pesticides through field soils - a review. Journal Environmental Quality, v.25, p.25-45, 1996.

23 FREIRE, J. C.; RIBEIRO, M. A. V.; BAHIA, V. G.; LOPES, A. S.; AQUINO, L. H. de. Resposta do milho cultivado em casade-vegetação a níveis de água em solo da região de Lavras (MG). Revista Brasileira de Ciência do Solo, v.4, p. 5-8, 1980.

24 GUERREIRO, M. C.; PEREIRA, E. L.; GONÇALVES, M. Avaliação da movimentação e degradação de atrazina em Latossolo Vermelho distrófico (LVd). In: REUNIÃO ANUAL DA SOCIEDADE BRASILEIRA DE QUÍMICA, 29., 2006, Águas de Lindóia. Anais... Águas de Lindóia: Sociedade Brasileira de Química, 2006.

25 HSBD. Hazardous Substance Data Bank. TOXNET toxicology data network. Disponível em: <http:// toxnet.nlm.nih.gov/cgi-bin/sis/search/f?./temp/ uw9SJ8:1>. Acesso em: 20 maio 2006.

26 HUANG, L. Q.; FRINK, C. R. Distribution of atrazine, simazine, alachlor, and metolachlor in soil profiles in Connecticut. Bulletim Environmental Contamination Toxicology, v.43, p.159-164, 1989.

27 JAVARONI, R. de C. A.; LANDGRAF, M. D.; REZENDE, M. O. O. Comportamento dos herbicidas atrazina e alaclor aplicados em solo preparado para o cultivo de cana de açúcar. Química Nova, v. 22, v.1, p.58-64, 1999.

28 KEMPER, W. D.; ROSENAU, R. C. Aggregate stability and size distribution. In: KLUTE, A. Methods of soil analysis, Part 1. Physical and mineralogical methods. $2^{\text {nd }}$ ed. Madison: American Society of Agronomy, 1986. p.425-441. (Agronomy Monograph, 9). 
29 LAABS, V.; AMELUNG, W.; PINTO, A.; ALTSTAEDT, A.; ZECH, W. Leaching and degradation of corn and soybean pesticides in an Oxisol of the Brazilian Cerrados. Chemosphere, v.41, p.1441-1449, 2000.

30 LAABS, V.; AMELUNG, W.; PINTO, A.; ZECH, W. Fate of pesticides in tropical soils of Brazil under field conditions. Journal Environment Quality, v.31, p.256-268, 2002.

31 LANGENBACH, T.; SCHROLL, R.; PAIM, S. Fate and distribution of ${ }^{14} \mathrm{C}$-atrazine in tropical soils. Chemosphere, v.40, p.449-455, 2000.

32 LAVORENTI, A.; PRATA, F.; REGITANO, J. B. Comportamento de pesticidas em solos: fundamentos. In: CURI, N.; MARQUES, J. J.; GUILHERME, L. R. G.; LIMA, J. M.; LOPES, A. S.; ALVAREZ V., V. H. (Org.). Tópicos em ciência do solo. Viçosa: SBCS, 2003. v.3, p.335-400.

33 LIMA, D. M. de. Sorção e deslocamento miscível da atrazina em amostras de Latossolos. Lavras, 2004.66 p. Dissertação (Mestrado em Agronomia - Solos e Nutrição de Plantas), Universidade Federal de Lavras.

34 LINDSAY, W. L. Chemical equilibria in soils. New York: Wiley-Interscience, 1979. 447 p.

35 LOPES, A. S. Solos sob "cerrado": características, propriedades e manejo. Piracicaba: Associação Brasileira para a Pesquisa da Potassa e do Fosfato, 1983. 162 p

36 LOPES, N. P. Adsorção do triadimenol por frações de solo associadas a um composto de lixo urbano. Viçosa, 1999. 100 p. Dissertação (Mestrado em Solos e Nutrição de Plantas), Universidade Federal de Viçosa.

37 MACIEL, G. Avaliação do movimento do herbicida simazina em colunas de solo LV (Latossolo Vermelho Escuro). Lavras, 2002. 67 p. Dissertação (Mestrado em Agroquímica e Agrobioquímica), Universidade Federal de Lavras.

38 MARQUES, J. J. G. S. M.; CURI, N.; LIMA, J. M. Recursos ambientais do Alto Rio Grande, Minas Gerais: problemas, fisiografia e guia de excursão técnica. Lavras: UFLA/FAEPE, 2002. 35 p.

39 MARTIN-NETO, L.; VIEIRA, E. N.; SPOSITO, G. Mechanism of atrazine sorption by humic acid: a spectroscopy study. Environmental Science and Technology, New York, v.28, p.1867-1873, 1994.

40 MATOS, L. M. de; SILVA, E. F. da. Influência das propriedades dos solos e de pesticidas no potencial de contaminação de solos e águas subterrâneas. Pesticidas: revista de ecotoxicologia e meio ambiente, Curitiba, v.9, p.103-124, 1999.

41 MIELNICZUK, J.; BAYER, C.; VEZZANI, F. M.; LOVATO, T.; FERNANDES, F. F.; DEBARBA, L. Manejo de solo e culturas e sua relação com os estoques de carbono e nitrogênio do solo. Tópicos em Ciência do Solo, Viçosa, v.3, p.209-248, 2003.

42 MOREAU, C.; MOUVET, C. Adsorption and desorption of atrazyne, deethylatrazyne and hydroxyatrazine by soil and aquifer solids. Journal of Environmental Quality, v.26, p.416-424, 1998.

43 NAKAGAWA, L. E.; ANDRÉA, M. M. Liberação de resíduos ligados de atrazina em solos por meio de esterilização por autoclavagem e radiação gama. Revista Brasileira de Ciência do Solo, v.21, p.707-710, 1997.

44 NAKAGAWA, L. E.; ANDRÉA, M. M. Degradação e formação de resíduos não-extraíveis ou ligados do herbicida atrazina em solo. Pesquisa Agropecuária Brasileira, v.35, n.8, p.1509-1515, 2000.

45 NÓBREGA, J. C. A. Calagem e fosfatagem na retenção e mobilidade do fungicida triadimenol em amostras de Latossolos. Lavras, 2004. 96 p. Tese (Doutorado em Agronomia - Solos e Nutrição de Plantas), Universidade Federal de Lavras.

46 OLIVEIRA JÚNIOR, R. S. de. Relação entre propriedades químicas e físicas do solo e sorção, dessorção e potencial de lixiviação de herbicidas. Viçosa, 1998. 83 p. Tese (Doutorado em Fitotecnia), Universidade Federal de Viçosa.

47 OLSEN, S. R.; WATANABE, F. S. A method to determine a phosphorous adsorption maximum of soils as measured by the Langmuir isotherm. Soil Science Society of America Proceedings, Madison, v.21, p.144-149, 1957.

48 PARAÍBA, L. C.; CERDEIRA, A. L.; SILVA, E. F.; MARTINS, J. S.; COUTINHO, H. L. C. Evaluation of soil temperature effect on herbicide leaching potential into groundwater in the Brazilian Cerrado. Chemosphere, v.53, p. 1087-1095, 2003.

49 PARK, J. K.; FENG, Y.; JI, P.; VOICE, T. C.; BOYD, S. A. Assessment of bioavailability of soil-sorbed atrazine. Applied Environmental Microbiology, v.69, n.6, p. 3288-3298, 2003.

50 PAVAN, M. A.; BINGHAM, F. T.; PRATT, P. F. Chemical and mineralogical characteristics of selected soils of the State of Parana, Brazil. Turrialba, Costa Rica, v.35, n.2, p.131-139, 1985.

51 PEIXOTO, M. de F. da S. P.; LAVORENTI, A.; REGITANO, J. B.; TORNISIELO, V. L. Degradação e formação de resíduos ligados de ${ }^{14} \mathrm{C}$-atrazina em Latossolo Vermelho Escuro e Glei Húmico. Scientia Agrícola, Piracicaba, v.57, n.1, p.117122,2000 
52 PEIXOTO, M. de F. da S. P.; LAVORENTI, A.; REGITANO, J. B.; TORNISIELO, V. L.; PEIXOTO, C. P.; SAMPAIO, L. S. de V.; SAMPAIO, H. S. de V. Remobilização de resíduos ligados de ${ }^{14} \mathrm{C}$-atrazina em ácidos fúlvicos. Ciência Rural, Santa Maria, v.35, n.2, p.340-346, 2005.

53 PLAKAS, K. V.; KARABELAS, A. J.; WINTGENS, T.; MELIN, T. A study of selected herbicides retention by nanofiltration membranes: the role of organic fouling. Journal of Membrane Science, v.284, p.291-300, 2006.

54 PRATA, F. Comportamento do glifosato no solo e deslocamento miscível de atrazina. Piracicaba, 2002. 149 p. Tese (Doutorado em Agronomia - Solos e Nutrição de Plantas), Universidade de São Paulo.

55 RODRIGUES, B. N.; ALMEIDA, F. S. de. Guia de pesticidas. 4.ed. Londrina: IAPAR, 1998. 648 p.

56 RODRIGUES, R. de S. O. Avaliação do nível de resíduos de atrazine e simazine em água, em áreas de cultivo intensivo de milho do Brasil. São Paulo, 2001. 63 p. Tese (Doutorado em Ecologia), Universidade de São Paulo.

57 TOMITA, R. Y.; BEYRUTH, Z.; SÁFADI, R. S.; FONTES, A. F. A. Toxicidade da mistura dos herbicidas atrazina e diuron sobre Daphnia similis (Crustacea, Cladocera). Arquivos do Instituto Biológico, v.71 (suplemento), p.708-710, 2004.

58 URZEDO, A. P. F. M. de. Sorção e degradação do inseticida thiamethoxam em amostras de solos na região de Lavras-MG. Lavras, 2004. 50 p. Dissertação (Mestrado em Agronomia), Universidade Federal de Lavras.

59 URZEDO, A. P. F. M. de; RIGITANO, R. L. de O.; LIMA, J. M. de; CASTRO, N. R. do A. Sorção do inseticida tiametoxam em amostras de solos da região de Lavras-MG. Pesticidas: revista de ecotoxicologia e meio ambiente, Curitiba, v.16, p.71-80, 2006.

60 VASCONCELOS, F. C. W. Transporte de atrazina influenciado por calagem e adubação fosfatada em ambientes de Latossolos da Bacia do Rio das Mortes (MG). Lavras, 2007. 119 p. Tese (Doutorado em Ciência do Solo), Universidade Federal de Lavras.

61 YING, G. G.; KOOKANA, R. S.; MALLAVARPU, M. Release behavior of triazine residues in stabilised contaminated soils. Environmental Pollution, v.134, p.71-77, 2005. 